\title{
A Child with Ambiguous Genitalia: An Atypical Presentation
}

\author{
Waqas Hanif Sheikh1, Naveed Asif1, Muhammad Younas², Nida Basharat ${ }^{1}$, Qurat-ul-Ain ${ }^{1}$ and Noreen ${ }^{1}$
}

\begin{abstract}
Lipoid congenital adrenal hyperplasia (LCAH) (OMIM No. 201710) is the most severe type of congenital adrenal hyperplasia $(\mathrm{CAH})$. Its clinical presentation includes lethal disturbance of adrenal and gonadal steroid synthesis due to impairment in the conversion of cholesterol to pregnenolone. Infants with this disorder experience salt loss, and glucocorticoid and mineralocorticoid deficiencies. Replacement therapy has enabled long-term survival. Classic LCAH is relatively common in Japan and Korea but extremely rare in Caucasian populations. An XY male 5-year-old child presented at Endocrine Clinic of Armed Forces Institute of Pathology with ambiguous genitalia and hyperpigmentation. He had family history of $\mathrm{CAH}$. His laboratory investigations revealed normal serum cortisol and $17 \mathrm{Hydroxy}(17 \mathrm{OH})$ progesterone levels with high plasma ACTH and renin levels. He had low aldosterone with inadequate response with hCG stimulation test. This is the first case of non-classic LCAH reported in the Pakistani population. Steroidogenic acute regulatory protein (StAR) gene mutations result in LCAH and the condition should be considered in the differential diagnosis of an XY child with primary adrenal insufficiency.
\end{abstract}

Key Words: Congenital adrenal hyperplasia, Steroidogenic acute regulatory protein, Adrenal insufficiency, Infant.

\section{INTRODUCTION}

Lipoid congenital adrenal hyperplasia (LCAH) is an autosomal recessive disorder, and one of the lethal types of congenital adrenal hyperplasia (CAH). It is characterised by impairment in gonadal and adrenal steroid hormones synthesis. ${ }^{1}$ Basic defect is mutation in steroidogenic acute regulatory (StAR) gene, which leads to defective StAR protein. Physiologically, StAR protein is expressed in gonads and adrenal glands. Mutation in StAR gene leads to defects in the movement of cholesterol through the mitochondrial membrane. In this way, cholesterol is not available for conversion into pregnenolone by desmolase (side chain cleavage) enzyme (P450scc), the first step in steroid hormones synthesis inside mitochondria. As a result, there is defect in the conversion of cholesterol to pregnenolone, which is precursor of mineralocorticoids, glucocorticoids, and sex steroids. ${ }^{2}$ Severe deficiency or insufficiency of adrenocortical hormones can lead to Addisonian crisis, an entity with salt loss. Defect in sex hormone synthesis can lead to impairment of sex development.

LCAH typically presents with ambiguous genitalia and Addison disease. Other conditions like deficiencies of 17 alpha hydroxylase (17OHD), 3b-hydroxysteroid dehydrogenase (3b-HSD), and cytochrome P450 oxidoreductase (PORD), should be considered in the differential diagnosis. ${ }^{3}$

1 Department of Chemical Pathology, Armed Forces Institute of Pathology (AFIP), Rawalpindi, Pakistan

2 Department of Central Laboratory, Combined Military Hospital (CMH), Multan, Pakistan

Correspondence: Dr. Waqas Hanif Sheikh, Department of Chemical Pathology, Armed Forces Institute of Pathology (AFIP), Rawalpindi, Pakistan

E-mail:drwaqas10@yahoo.com

Received: October 11, 2018; Accepted: February 01, 2019
Herein, we report a boy with non-classic LCAH presenting to Endocrine Clinic of Armed Forces Institute of Pathology.

\section{CASE REPORT}

A 5-year boy, resident of Taxila, presented with complaints of ambiguous genitalia since birth and progressive weakness for 2 years. A few days after birth, parents brought him to local doctor who told that child was alright. Three years later, child started developing hyperpigmentation all over the body, more so on hands and face. At the age of 4 years, he started having vomiting and dehydration. One of his sisters died at 6 months of age due to $\mathrm{CAH}$ and one brother is a known case of $\mathrm{CAH}$.

The child was delivered by Caesarian-section with an APGAR score of $8 / 10$. His height and weight were more than $70^{\text {th }}$ percentile. Genital examination revealed a small phallus, poorly formed scrotum, bilateral undescended testes and bilateral inguinal hernia. Systemic examination was unremarkable.

His baseline laboratory investigations revealed decreased hemoglobin level with normal total leucocyte count and normal renal function tests, liver function tests and electrolytes, except for mild hyponatremia. Low normal level of serum dehydroepiandrosterone (DHEAS) and serum $17 \mathrm{OHP}$ ruled out 3 beta hydroxysteroid dehydrogenase deficiency. hCG stimulation test was performed, which showed inadequate response ruling out AIS and 5 alpha reductase deficiency. Serum TSH was within normal limits, whereas serum cortisol was at lower normal limit. Aldosterone was low with raised plasma ACTH and plasma renin. Karyotype was $46 \mathrm{XY}$. Ultrasonography revealed testes in inguinal area bilaterally and there was no evidence of female genital tract remnants. All these findings were consistent with LCAH. 
The child was started on treatment with Tab hydrocortisone, $20 \mathrm{mg}, 1 / 2$ twice a day and Tab florinef, $0.1 \mathrm{mg}$ once a day. The consultant endocrinologist advised to raise him as a girl. But parents insisted to raise him as a boy and bilateral orchidopexy was performed.

\section{DISCUSSION}

In this case report, we presented the complete medical history, examination, and management of a child who was diagnosed as LCAH. This child presented with atypical clinical features of adrenal insufficiency, such as mild hyponatremia and mild hyperpigmentation, and had ambiguous genitalia despite male karyotype. ${ }^{4}$

LCAH can easily be differentiated from CAH due to 21 hydroxylase deficiency. In 21-hydroxylase deficiency $\mathrm{CAH}$, karyotype $\mathrm{XX}$ child presents with ambiguous genitalia while $X Y$ presents with normal male type genitalia. However, in LCAH, XX child presents with normal genitalia but $X Y$ presents with ambiguous or female like genitalia. ${ }^{5}$

LCAH can be differentiated from other types of CAH by analysing the level of various serum steroid metabolites. Raised level of various steroid metabolites can be found in different forms of $\mathrm{CAH}$, but are not found in LCAH, e.g. patients with 21-hydroxylase deficiency $\mathrm{CAH}$ present with excessively high levels of 17-hydroxyprogesterone (17 OHP) and DHEA, while 17-delta-5-hydroxy-pregnenolone are found in 3-beta hydroxy steroid dehydrogenase (3b-HSD) deficiency. ${ }^{6}$

In spite of its rarity, it is now well established that StAR gene mutations lead to LCAH. About 40 StAR gene mutations have been identified; and 190 patients diagnosed with LCAH have been described in literature worldwide. In literature, the reported cases were siblings of patients with LCAH, or they were born from parents with consanguineous marriages. In spite of its very low prevalence, majority of cases of LCAH have been reported in Japanese, Korean, and Palestinian populations, but are rarely reported elsewhere. . $^{7}$

Although Huang et al. first described LCAH and StAR mutations in 10 Chinese children, there are no statistics on prevalence and incidence among Chinese population in literature. ${ }^{10}$ Physiologically, StAR protein is characterised by a highly specified amino acid sequence (from amino acids 67 to 280), which serves as a transporting module by binding cholesterol, called StAR-related lipid transfer domain. Mutations in StAR gene lead to impaired function of this StAR protein, leading to severely disturbed steroid synthesis. In the study that included 10 Chinese children diagnosed as LCAH, this variant gene accounted for $15 \%$ of the total mutant alleles with one parent being a carrier. ${ }^{10}$
In this patient, there was evidence of inherited mutation and parental consanguinity, leading to possibility of StAR gene heterozygous mutations. Prevalence of StAR gene mutations is more in some areas, but not restricted to those geographical regions. Karyotype $46 \mathrm{XY}$ with phenotype of female external genitalia is more likely to be diagnosis because of lack of Mullerian ducts, as documented by pelvic ultrasonography. Karyotype 46XX females have the same prevalence of this autosomal recessive disease. Even today, based on the absence of disorder of sex development (DSD), karyotypically females are not analysed for defects involving gonadal and adrenal gland steroid hormones synthesis and they may be considered as idiopathic adrenal insufficiency.

In conclusion, StAR gene mutations leading to classic LCAH should be considered in the differential diagnosis with $X Y$ karyotype presenting with ambiguous genitalia in children.

\section{REFERENCES}

1. King SR, Bhangoo A, Stocco DM. Functional and physiological consequences of StAR deficiency: Role in lipoid congenital adrenal hyperplasia. Endocr Dev 2011; 20:47-53.

2. Kaur J, Casas L, Bose HS. Lipoid congenital adrenal hyperplasia due to StAR mutations in a Caucasian patient. Endocrinol Diabetes Metab Case Rep 2016; 2016:150119.

3. Gassner HL, Toppari J, Quinteiro Gonzalez S. Near-miss apparent SIDS from adrenal crisis. J Pediatr 2004; 145:178-83.

4. Miller WL, Bose HS. Early steps in steroidogenesis: Intracellular cholesterol trafficking. J Lipid Res 2011; 52:2111-35.

5. White PC, Speiser PW. Congenital adrenal hyperplasia due to 21-hydroxylase deficiency. Endocr Rev 2000; 21:245-91.

6. Yuksel B, Kulle AE, Gurbuz F. The novel mutation p.Trp147Arg of the steroidogenic acute regulatory protein causes classic lipoid congenital adrenal hyperplasia with adrenal insufficiency and 46,XY disorder of sex development. Horm Res Paediatr 2013; 80:163-9.

7. Miller WL. Congenital lipoid adrenal hyperplasia: The human gene knockout of the steroidogenic acute regulatory protein. J Mol Endocrinol 1997; 19:227-40.

8. Lin D, Sugawara T, Strauss JF III, Clark BJ, Stocco DM, Saenger $\mathrm{P}$, et al. Role of steroidogenic acute regulatory protein in adrenal and gonadal steroidogenesis. Science 1995; 267:1828-31.

9. Camats N, Pandey AV, Fernández-Cancio M, Fernández JM, Ortega AM, Udhane $\mathrm{S}$, et al. StAR splicing mutations cause severe phenotype of lipoid congenital adrenal hyperplasia: Insights from a novel splice mutation and review of reported cases. Clin Endocrinol (Oxf) 2014; 80:191-9.

10. Huang Z, Ye J, Han L, Qu W, Zhang H, Yu Y, et al. Identification of five novel STAR variants in ten Chinese patients with congenital lipoid adrenal hyperplasia. Steroids 2016; 108: 85-91. 